\title{
甲磺酸培氟沙星与人血清白蛋白之间结合模式的研究 *
}

\author{
马国正 ${ }^{1}$ 谭 非 1 蒋勇军 ${ }^{2}$ 郑柯文 ${ }^{1}$ 郭 明 1 俞庆森 ${ }^{1,2}$ \\ $\left({ }^{1}\right.$ 浙江大学理学院化学系, 杭州 310027 ; \\ 2 浙江大学宁波理工学院药物分子设计与营养工程重点实验室, 宁波 315100)
}

\begin{abstract}
摘要 采用实验和计算的方法研究了甲磺酸培氟沙星与人血清白蛋白之间的结合作用. 苂光法测得甲磺酸培 氟沙星与人血清白蛋白形成一种类型的复合物, 结合常数为 $1.7 \times 10^{5} \mathrm{~L} \cdot \mathrm{mol}^{-1}$, 有 1.05 个平均结合位点; 微量 热法测得该药物 - 蛋白结合过程中焓变为 $1.03 \mathrm{~kJ} \cdot \mathrm{mol}^{-1}$, 滳变为 $101.28 \mathrm{~J} \cdot \mathrm{K}^{-1} \cdot \mathrm{mol}^{-1}$, 反应为熵驱动. 用分 子对接的方法预测了甲磺酸培氟沙星与人血清白蛋白的结合模式. 计算表明, 甲磺酸培氟沙星可结合在人血清 白蛋白的两个药物结合位点, 疏水作用即熵效应在药物与蛋白的结合中起重要作用, 预测的结合自由能和实验 值基本一致。
\end{abstract}

关键词: 甲磺酸培氟沙星, 人血清白蛋白, 分子模建, 结合模式 中图分类号: 0641

人血清白蛋白 (HSA) 是一种含有 585 个氨基 酸残基的球蛋白, 在人体血浆中浓度大约为 $40 \mathrm{~g} \cdot$ $\mathrm{L}^{-1}$, 占血浆蛋白总量的 $60 \%$ 左右. HSA 作为人体 血液循环系统中一种主要的运输蛋白, 可广泛结合 一些内源性和外源性的配体, 诸如不饱和脂肪酸、 胆红素、胆酸等,以方便它们在循环系统中的运输 . 此外, HSA 还可以结合多种药物分子、代谢物和有 机物。药物和 HSA 的结合很大程度上影响到药物 在体内的吸收和消除, 对药物和 HSA 结合模式的 研究能够提供有效的结构信息, 在确定药物的有效 治疗浓度以及药物代谢动力学等方面有积极意义.

通过 $\mathrm{X}$ 射线衍射得到了 HSA 高分辨率的三维 晶体结构, 它包含三个结构相似的 $\alpha$ 螺旋结构域 (Domain I, II, III)，每个结构域又包含两个结构子 域 (Subdomain A, B), 分别由 6 个 $\alpha$ 螺旋和 4 个 $\alpha$ 螺旋组成。脂肪酸的结合位点分布于 HSA 所有的 6 个子域, 而药物分子主要结合在 Subdomain IIA 和 IIIA, 即 Sudlow's sites I 和 II. 虽然药物与 HSA 的 结合模式前人已经进行了大量的研究, 有些还报道 了晶体结构, 但由于 HSA 结合药物的广泛性和复 杂性, 详细具体的药物结合位点仍然未知 ${ }^{[1-6]}$.

喹诺酮类是含有 4- 喹诺酮母核基本结构的合
成抗菌素, 具有抗菌广谱、强活性、低毒副作用及临 床高效等特点, 到目前已经发展到第三代。喹诺酮 药物和蛋白的相互作用前人已经做了很多工作, 但 大都局限在实验方面 ${ }^{[7-9]}$, 并没有从计算角度来预测 药物与蛋白的结合模式以及合理解释药物与蛋白 之间的结合作用。本文从实验结合计算的角度对 甲磺酸培氟沙星 (PFLX) 与 HSA 的结合模式进行了 研究, 运用分子模建的方法来预测了药物与蛋白结 合的具体作用位点和详细的作用机理 .

\section{1 实验部分}

\section{1 仪器与试剂}

日立 F-4500 菼光仪, UV-260 紫外分光光度计, 瑞典产 LKB-2277 型微量热仪. PFLX 为纯品原料 药, HSA(98\%, Sigma 公司), 三差甲基氨基甲烷 (Tris) 为生化试剂, 盐酸等均为分析纯试剂, 实验用 水为亚沸蒸馏去离子水。配制浓度为 $0.05 \mathrm{~mol}$. $\mathrm{L}^{-1}, \mathrm{pH}=7.0$ 的 Tris- $\mathrm{HCl}$ 缓冲溶液 (加 $0.10 \mathrm{~mol} \cdot$ $\mathrm{L}^{-1}$ 的 $\mathrm{NaCl}$ 溶液以维持离子强度), 用此缓冲溶液 进一步配制 HSA 及药物等梡备溶液.

\section{2 实验方法}

苂光光谱测定: 准确移取 $2.5 \mathrm{~mL}$ 浓度为 1.0 
$\mathrm{mmol} \cdot \mathrm{L}^{-1}$ 的 $\mathrm{HSA}$ 溶液于 $1 \mathrm{~cm}$ 的石英比色血.中, 用微量注射器逐次加人 $1.0 \mathrm{mmol} \cdot \mathrm{L}^{-1}$ 的 PFLX 溶 液进行苂光滴定 (滴定剂累加体积 $<100 \mu \mathrm{L}$ ), 测定 时苂光发射与激发狭缝宽度均为 $5 \mathrm{~nm}$, 波长扫描速 率为 $240 \mathrm{~nm} \cdot \mathrm{s}^{-1}$, 固定激发波长 $285 \mathrm{~nm}$.

微量热测定: 采用流动混合量热方式, 以缓冲 溶液确定基线, 将已知浓度的 HSA 和 PFLX 溶液以 相同流速泵人 LKB-2277 微量热计的混合流动池 中, 读取系统达到稳定后的热功率, 同时测定相同 条件下药物溶液以及 HSA 溶液的稀释效应。量热 计标定采用电能标定法, 实际流速用重量法测定.

\section{3 分子模建}

分子模建采用 SGI O2 工作站上的 Dock 4.02 程序包计算, Dock 能够给出配体位于受体活性位点 处合理的取向和构象. 配体 PFLX 结构来源于剑桥 晶体数据库 (CSD), 受体蛋白 HSA 取自蛋白质晶体 数据库 (PDB). 分子模建按照以下步骤进行: (i) 确 定潜在的 PFLX 结合位点; (ii) 在配体结合位点填充 球簇以映射受体结合腔穴的性质; (iii) 尝试匹配不 同小球中心的距离和配体中不同原子的距离, 进行 配体在受体活性位点处的构象搜索; (iv)对配体的 不同取向进行定位, 以经验势能函数作为评价函 数, 找到配体与受体的最佳结合方式.

\section{2 结果与讨论}

\section{1 荧光法测定 PFLX 与 HSA 相互作用的结合 常数和结合位点数}

为了研究 PFLX 和 HSA 相互作用的情况, 我们 进行了苂光淬灭的实验测定. 取浓度皆为 $1.0 \times$ $10^{-5} \mathrm{~mol} \cdot \mathrm{L}^{-1}$ 的 PFLX、HSA 以及两者等物质量混 合的溶液体系进行苂光测定 (图 1). 此外, 在 $1.0 \times$

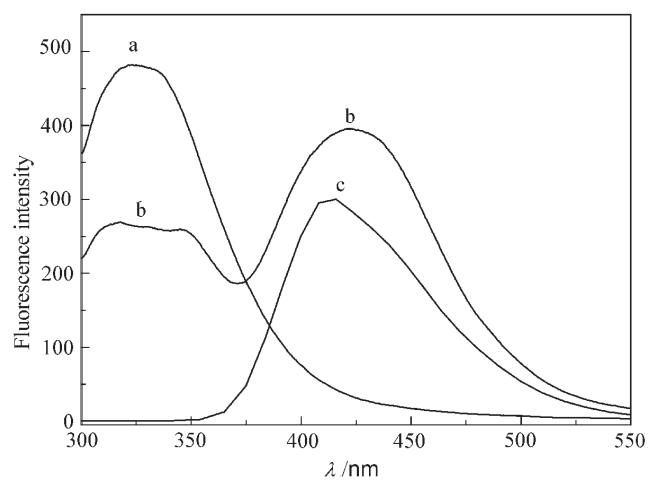

图 1 各试样的荧光光谱

Fig. 1 Fluorescence emission spectra of the samples $\lambda_{\text {ex }}=285 \mathrm{~nm}, \mathrm{pH}=7.0$; a) HSA, b) PFLX + HSA (1:1), c) PFLX

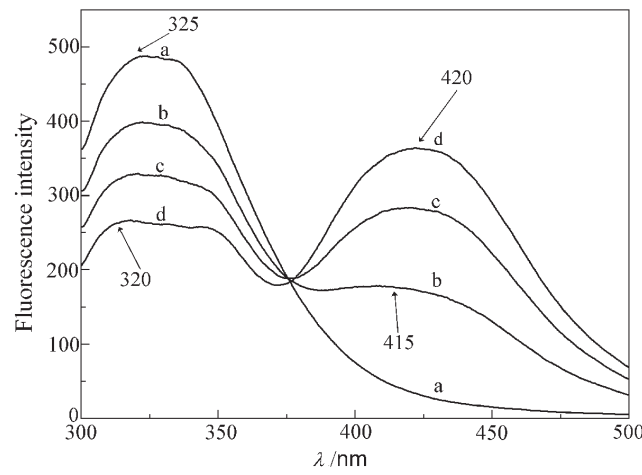

图 2 甲磺酸培氟沙星对人血清白蛋白荧光光谱的影响

Fig. 2 Effect of PFLX on fluorescence spectra of HSA

[HSA]: $1.0 \times 10^{-5} \mathrm{~mol} \cdot \mathrm{L}^{-1} ; \lambda_{\text {ex }}=285 \mathrm{~nm} ; \mathrm{pH}=7.0$; $10^{5}[\mathrm{PFLX}] / \mathrm{mol} \cdot \mathrm{L}^{-1}$ : a) 0, b) 0.4 , c) 0.8 , d) 1.2

$10^{-5} \mathrm{~mol} \cdot \mathrm{L}^{-1}$ 的 HSA 溶液中依次加人不同浓度的 PFLX 溶液进行苂光测定, 以此探讨 PFLX 与 HSA 结合在不同浓度配比情况下的菼光效应(图 2).

由图 2 可以看出, PFLX 的加人使 HSA 的荧光 强度降低, 发射波长由 $325 \mathrm{~nm}$ 移至 $320 \mathrm{~nm}$, 而 PFLX 由于 HSA 的加人苂光强度增加, 最大发射波 长由 $415 \mathrm{~nm}$ 移至 $420 \mathrm{~nm}$. 由此可以说明 $\mathrm{HSA}$ 与 PFLX 之间有能量转移, 能够产生较强的相互作用.

在药物 - 蛋白结合的研究中, 对结合常数的计 算有几种不同的计算方法, 其中最常用的是 Scatchard 方程 ${ }^{[10]}$ :

$$
\nu /[\mathrm{D}]=-K \nu+K n
$$

$\nu$ 为平均结合数, 即平均每摩尔大分子结合药物小 分子的物质的量; $K$ 为结合常数; $n$ 为结合位点数, 即一个大分子能结合小分子的数目; [D]为溶液中 药物的游离浓度. 在所考虑的波长范围, 若体系的 苂光仅由 HSA 所产生, 则有:

$$
F_{0} / F=[\mathrm{P}] /\left[\mathrm{P}_{\mathrm{t}}\right]
$$

按 $\nu$ 的定义, 应有:

$$
\begin{aligned}
\nu & =\left(\left[\mathrm{D}_{\mathrm{t}}\right]-[\mathrm{D}]\right) /\left[\mathrm{P}_{\mathrm{t}}\right] \\
& =n\left(\left[\mathrm{P}_{\mathrm{t}}\right]-[\mathrm{P}]\right) /\left[\mathrm{P}_{\mathrm{t}}\right] \\
& =n\left(F_{0}-F\right) / F_{0}
\end{aligned}
$$

$\left[\mathrm{D}_{\mathrm{t}}\right]$ 和 $\left[\mathrm{P}_{\mathrm{t}}\right]$ 分别为体系中药物与 HSA 的总浓度, $F$ 和 $F_{0}$ 分别表示加人和不加人药物时 HSA 溶液的 苂光强度. 结合公式 (1) 与 (3) 可以得到:

$$
F_{0} / F=K\left[D_{\mathrm{t}}\right] F_{0} /\left(F_{0}-F\right)-n K\left[\mathrm{P}_{\mathrm{t}}\right]
$$

固定 HSA 的浓度 $\left[\mathrm{P}_{\mathrm{t}}\right]$, 改变药物浓度 $\left[\mathrm{D}_{\mathrm{t}}\right]$, 按 公式 (4) 对实验数据做线性拟合即可确定结合常数 $K$ 和结合位点数 $n$, 如图 3 所示.

由公式(4)可求得 $n=1.05, K=1.7 \times 10^{5} \mathrm{~L}$. 


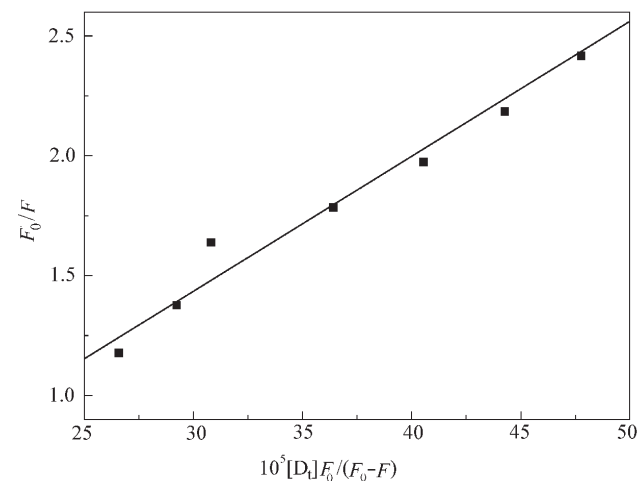

图 3 PFLX-HSA 溶液体系荧光强度变化的拟合结果

Fig. 3 Fitting curve of fluorescence intensity changes of the PFLX-HSA solution system

$\mathrm{mol}^{-1}$; 故可得知甲磺酸培氟沙星与人血清白蛋白约 有一个结合位点, 结合常数为 $1.7 \times 10^{5} \mathrm{~L} \cdot \mathrm{mol}^{-1}$.

\section{2 微量热测定蛋白与药物结合过程的焓变}

反应的热力学量 $\Delta_{\mathrm{r}} H_{\mathrm{m}}$ 和 $\Delta_{\mathrm{r}} S_{\mathrm{m}}$ 对于确定药物 蛋白的结合模式有重要意义, 因此, 我们用微量热法 求解焓变等热力学量, 从热力学角度来理解甲磺酸 培氟沙星与人血清白蛋白作用模式和作用机理 .

药物与蛋白通常表现为以范德华作用、静电作 用、氢键和疏水作用等形式的非键作用结合在一起, 这种非键作用常采用位点结合模式来描述，这是一 种经验模式, 可用多级结合方程表示:

$$
\nu=\frac{\left[\mathrm{D}_{\mathrm{b}}\right]}{\left[\mathrm{P}_{\mathrm{t}}\right]}=\frac{\left[\mathrm{D}_{\mathrm{t}}\right]-[\mathrm{D}]}{\left[\mathrm{P}_{\mathrm{t}}\right]}=\frac{n K_{\mathrm{A}}[D]}{1+K_{\mathrm{A}}[D]}
$$

$\left[\mathrm{D}_{\mathrm{b}}\right] 、[\mathrm{D}]$ 分别为药物的结合浓度和游离浓度, $\left[\mathrm{P}_{\mathrm{t}}\right]$ 为蛋白质总浓度, $\nu$ 为平均结合数, $n$ 和 $K_{\mathrm{A}}$ 分别表 示结合位点数和结合常数.

由 (5)式变换得到:

$$
\frac{1}{\nu}=\frac{1}{n}+\frac{1}{n K_{\mathrm{A}}[\mathrm{D}]}
$$

由苂光法可求得 $n$ 和 $K_{\mathrm{A}}$, 通过 (5) 式可推算出:

$\left[\mathrm{D}_{\mathrm{t}}\right]-[\mathrm{D}]=0.5\left(1 / K_{\mathrm{A}}+n\left[\mathrm{P}_{\mathrm{t}}\right]+\left[\mathrm{D}_{\mathrm{t}}\right]\right)-$

$0.5\left(\left(1 / K_{\mathrm{A}}+n\left[\mathrm{P}_{\mathrm{t}}\right]+\left[\mathrm{D}_{\mathrm{t}}\right]\right)^{2}-4 n\left[\mathrm{P}_{\mathrm{t}}\right]\left[\mathrm{D}_{\mathrm{t}}\right]\right)^{1 / 2}$

\section{表 1 PFLX 结合 HSA 的热力学参数}

Table 1 The thermodynamic parameters for the binding of

\begin{tabular}{|c|c|c|c|}
\hline & $\Delta_{\mathrm{r}} H_{\mathrm{m}} / \mathrm{kJ} \cdot \mathrm{mol}^{-1}$ & $\Delta_{\mathrm{r}} G_{\mathrm{m}} / \mathrm{kJ} \cdot \mathrm{mol}^{-1}$ & $\Delta_{\mathrm{r}} S_{\mathrm{m}} / \mathrm{J} \cdot \mathrm{K}^{-1} \cdot \mathrm{mol}^{-1}$ \\
\hline & 1.01 & & 103. 40 \\
\hline & 1. 04 & & 99.14 \\
\hline & 1. 04 & & 101.30 \\
\hline Average & 1. 03 & -29.85 & 101. 28 \\
\hline
\end{tabular}
PFLX to HSA
$\left[D_{t}\right]$ 已知, 从而可以求得 $[D]$, 由 (6) 式可求出 对应的 $\nu$ 值. 因此, 根据流动混合量热方式, 可求 得以下几个热力学量 (见表 1 ):

$$
\begin{aligned}
& \Delta_{r} \mathrm{H}_{\mathrm{m}}= \pm Q /\left(\nu f_{\mathrm{p}}\left[\mathrm{P}_{\mathrm{t}}\right]\right) \\
& \Delta_{r} G_{\mathrm{m}}=-R T \ln K_{\mathrm{A}} \\
& \Delta_{\mathrm{r}} S_{\mathrm{m}}=\left(\Delta_{\mathrm{r}} S_{\mathrm{m}}-\Delta_{\mathrm{r}} G_{\mathrm{m}}\right) / T
\end{aligned}
$$

式中 “+”表示吸热反应，“””表示放热反应; $f_{\mathrm{p}}$ 表示 蛋白流速; $Q$ 为反应热功率 (即总热功率扣除各反 应物相同条件下稀释效应后的净热功率).

PFLX 与 HSA 结合过程 $\Delta_{\mathrm{r}} H_{\mathrm{m}}$ 很小, 可认为近似 等于零, 此反应为熵驱动. Subramanian 等 ${ }^{[11]}$ 的研究 表明, 在药物 - 蛋白结合过程中, 正的 $\Delta_{\mathrm{r}} S_{\mathrm{m}}$ 经常可 认为是存在疏水作用的证据. 因此, 我们可以认为, 在 PFLX-HSA 相互作用中, 疏水作用是此药物 - 蛋 白复合物形成的主要作用力。

\section{3 分子模建 PFLX 与 HSA 的结合}

许多研究表明, HSA 能在不同的结合位点结合 多种配体, 其中药物的结合位点主要有两个 (Sudlow's sites I 和 II). 我们分别对 PFLX 在 HSA 的 Sudlow's sites I 和 II 两个药物结合位点进行分子 对接的研究, HSA 晶体结构取自蛋白质晶体数据库 中 HSA 与 R-warfarin 的复合物晶体结构 (PDB 编号 $1 \mathrm{~h} 9 \mathrm{z}$ ). 对于 Sudlow's site I, 我们取 $1 \mathrm{~h} 9 \mathrm{z}$ 的 warfarin 结合口袋为活性位点, 对于 Sudlow's site II, 取 $1 \mathrm{~h} 9 \mathrm{z}$ 中脂肪酸结合口袋 FA3 和 FA4 为活性位点. 分子 对接结果表明 PFLX 可同时结合在两个药物结合位 点, 所得结合能值如表 2 所示, 在 Sudlow's sites I 为 $-24.18 \mathrm{~kJ} \cdot \mathrm{mol}^{-1}$, 在 Sudlow's site II 为 -25.89 $\mathrm{kJ} \cdot \mathrm{mol}^{-1}$, 与苂光法实验测定结合自由能 -29.85 $\mathrm{kJ} \cdot \mathrm{mol}^{-1}$ 基本一致.

DOCK 计算所得的分子内能可以大概估计出底 物结合受体时几何构型的扭曲程度, 较负的分子间 能和较正的分子内能可以说明底物有可能结合在受 体的表面 ${ }^{[12]}$. 由此, 从表 2 可以看出, 在 PFLX-HSA 复合物体系中, 可认为 PFLX 结合在 HSA 的表面活 性口袋处, 结合部位较浅。这点也可从图 $4 \mathrm{~b}$ 和图 $4 \mathrm{~d}$ 得到说明.

\section{表 2 PFLX 在 HSA 结构域 IIA 和 IIIA 结合位点处的结 合能 $\left(\mathbf{k J} \cdot \mathbf{m o l}^{-1}\right)$}

Table 2 Energies of binding sites for PFLX in domain IIA and IIIA of HSA $\left(\mathrm{kJ} \cdot \mathrm{mol}^{-1}\right)$

\begin{tabular}{lcccc}
\hline & \multicolumn{2}{c}{ Energy of Sudlow's site I } & \multicolumn{2}{c}{ Energy of Sudlow's site II } \\
\cline { 2 - 5 } & Intramolecular & Intermolecular & Intramolecular & Intermolecular \\
\hline PFLX & 5.55 & -29.72 & 6.06 & -31.95 \\
\hline
\end{tabular}



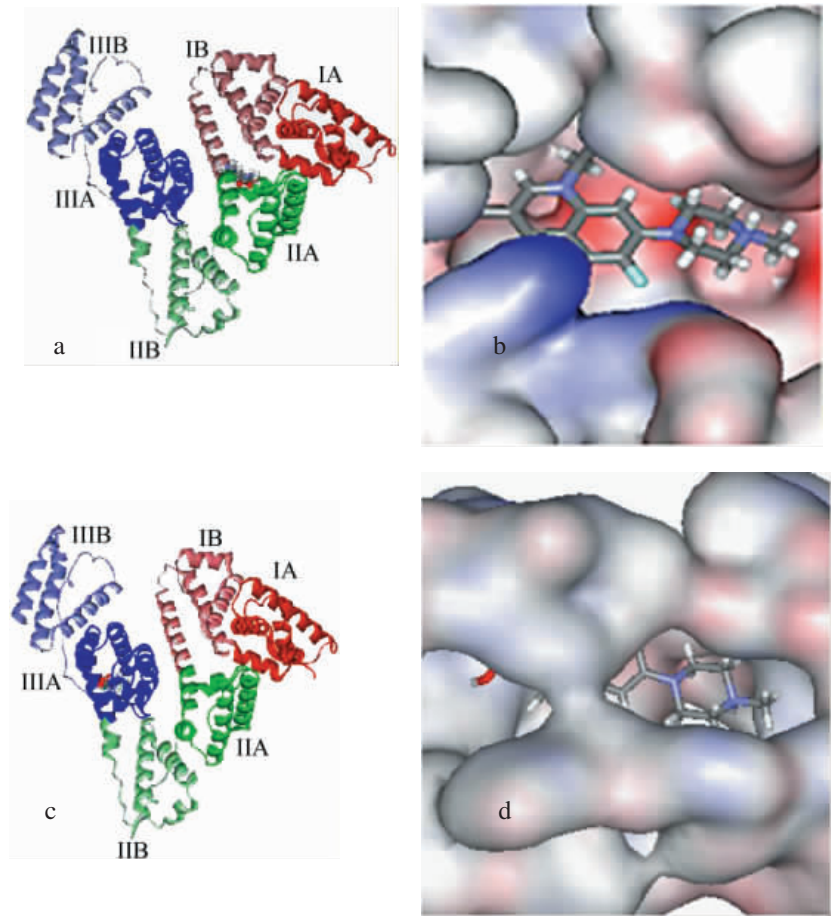

图 4 PFLX 与 HSA 的结合模式示意图

Fig. 4 The binding modes between PFLX and HSA a\&c, overview of the HSA-PFLX complex showing the drug binding to Sudlow's sites I and II. The protein secondary structure is shown schematically, and the domains are color-coded as follows: I, red; II, green; III, blue. The A and B subdomains are depicted in dark and light shades, respectively. PFLX are shown in a space-filling representation and colored by atom type (carbon, gray; oxygen, red; fluorine, green ). b\&d, side view of PFLX binding pocket of Sudlow's sites I and II. The figure was made with DS Modeling 1. 1.

分子对接所得到的两个结合位点数与菼光实 验测得的一个平均结合位点数并不矛盾, 这是因为 药物在这两个结合位点的结合能差别太小, 以至于 实验不能区分, 所以苂光实验掩盖了药物与蛋白的 结合位点数.

从图 4 PFLX 与 HSA 结合模式示意图可以看 出, PFLX 可以结合在 HSA 的 Sudlow's sites I 和 II, 结合部位较浅, 主要依靠疏水作用的结合. 图 5 为 PFLX 与 HSA 配体结合口袋周围的残基相互作用 效果图, 可以很清楚地看到 PFLX 结合位点处的疏 水相互作用.

从微量热实验得出, 疏水作用是药物和蛋白质结 合的主要驱动力, 这和前人的实验结果相一致 ${ }^{[13-14]}$. 分子模建结果也显示药物结合在蛋白质表面的疏 水口袋内, 疏水作用在 PFLX-HSA 复合物中起重要 的作用. 如图 5 所示, PFLX 在 HSA 的两个结合口

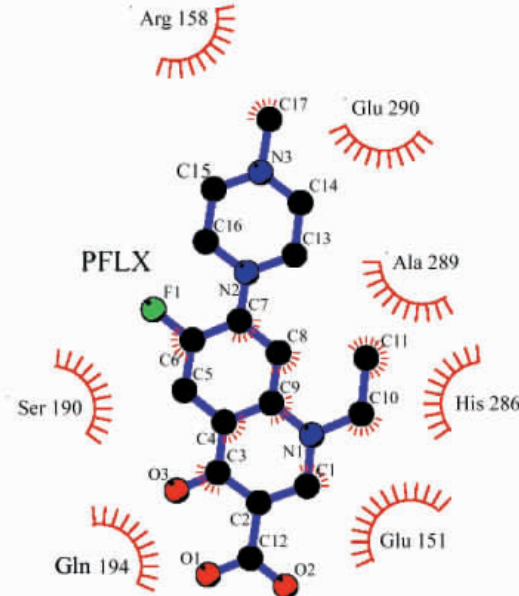

Sudlow's site I

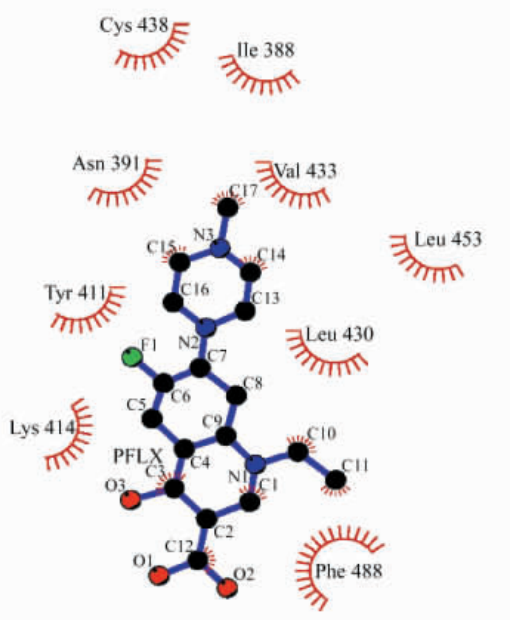

Sudlow's site II

图 5 PFLX 结合位点的疏水作用示意图

Fig. 5 Schematic diagrams of hydrophobic interactions between PFLX and HSA

Hydrophobic interactions are shown as spiked spheres (hydrophobic interactions with distances of less than 0.35 $\mathrm{nm}$ ). This figure was made with the program LIGPLOT.

袋周围主要与疏水残基相互作用, 在 Sudlow's site I, Glu151、Arg158、Ser190、Gln194、His 286、Ala289 和 Glu290 形成一个疏水区, 成为甲磺酸培氟沙星的一 个结合口袋; 在 Sudlow's site II, Ile388、Asn391、 Tyr411、Lys414、Leu430、Val433、Cys438、Leu453 和 Phe488 组成另外一个疏水结合口袋. 此处, 分子模 建的复合物模型还能为进一步研究如氨基酸残基的 突变实验提供一定的结构依据.

\section{3 结 论}

本文采用实验结合计算的方法研究了甲磺酸 培氟沙星与人血清白蛋白之间结合作用的情况, 通 
过苂光法和微量热法实验测定了 PFLX 与 HSA 的 结合位点数、结合常数以及焓变等热力学量.同 时, 利用分子对接的方法模建了 PFLX 与 HSA 受体 的结合模式, 计算出的结合能能较好地预测实验值. 说明 PFLX 可以结合在 HSA 的两个药物结合位点, 疏水作用起主导作用。

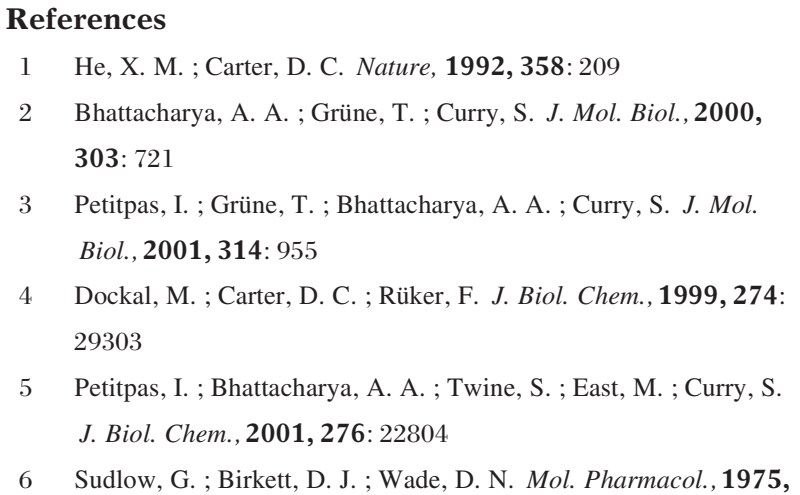

11: 824

7 Shen, L. L. ; Mitscher, L. A. ; Sharma, P. N. ; O'Donnell, T. J. ; Chu, D. W. T. ; Cooper C. S. ; Rosen T. ; Pernet, A. G. Biochemistry, 1989, 28: 3886

8 Permana, P. A. ; Snapka, R. M. ; Shen, L. L. ; Chu, D. T. W. ; Clement, J. J. ; Plattenr, J. J. Biochemistry, 1994, 33: 1333

9 Guo, M. ; Zou, J. W. ; Yi, P. G. ; Shang, Z. C. ; Hu, G. X. ; Yu, Q. S. Anal. Sci., 2004, 20: 465

10 Scatchard, G. Ann, N. Y. Acad. Sci., 1949, 51: 660

11 Ross, P. D. ; Subramanian, S. Biochemistry, 1981, 20: 3096

12 Shobini, J. ; Mishra, A. K. ; Sandhya, K. ; Chandra, N. Spectrochimica Acta Part A, 2001, 57: 1133

13 Shang, Z. C. ; Yi, P. G. ; Yu, Q. S. ; Lin, R. S. Acta Phys. -Chim. Sin., 2001, 17 (1): 48 [商志才, 易平贵, 俞庆森, 林瑞森. 物 理化学学报 (Wuli Huaxue Xuebao), 2001, 17 (1): 48]

14 Guo, M. ; Yi, P. G. ; Zhao, W. N. ; Shang, Z. C. ; Yu, Q. S. Journal of Zhejiang University (Science Edition), 2003, 30(4): 434 [ 郭 明, 易平贵, 赵文娜, 商志才, 俞庆森. 浙江大学学 报 (理学版) (Zhejiang Daxue Xuebao), 2003, 30 (4): 434]

\title{
The Binding Mode of Pefloxacin Mesylate with Human Serum Albumin*
}

\author{
MA, Guo-Zheng ${ }^{1} \quad$ TAN, Fei ${ }^{1} \quad$ JIANG, Yong-Jun ${ }^{2} \quad$ ZHENG, Ke-Wen ${ }^{1} \quad$ GUO, Ming ${ }^{1} \quad$ YU, Qing-Sen ${ }^{1,2}$ \\ ( ${ }^{1}$ Department of Chemistry, Zhejiang University, Hangzhou 310027; ${ }^{2}$ Key Laboratory for Molecular Design and Nutrition \\ Engineering of Ningbo City, Ningbo Institute of Technology, Zhejiang University, Ningbo 315100 )
}

\begin{abstract}
This study was designed to explore the binding mode of pefloxacin mesylate(PFLX) with human serum albumin (HSA). Fluorescence spectroscopic results showed pefloxacin-HSA complex with binding constants of $1.7 \times 10^{5} \mathrm{~L} \cdot \mathrm{mol}^{-1}$. The enthalpy change $\left(\Delta_{\mathrm{r}} H_{\mathrm{m}}\right)$ and entropy change $\left(\Delta_{\mathrm{r}} S_{\mathrm{m}}\right)$ were measured by microcalorimetry to be $1.03 \mathrm{~kJ} \cdot \mathrm{mol}^{-1}$ and $101.28 \mathrm{~J} \cdot \mathrm{K}^{-1} \cdot \mathrm{mol}^{-1}$, which indicated that hydrophobic interaction played a major role in the binding of PFLX with HSA. The possible binding domains of HSA for PFLX were studied by molecular modeling, which predicted that the hydrophobic interaction played a dominating role in this drug-protein interaction and PFLX could bind to Sudlow's sites I and II of HSA. The binding energies obtained from the molecular modeling were in accordance with the experiment results.
\end{abstract}

Keywords: Pefloxacin mesylate, Human serum albumin, Molecular modeling, Binding mode 